\title{
Automatic myocardium segmentation of LGE MRI by deformable models with prior shape data
}

\author{
YingLi Lu', Graham Wright ${ }^{1,2^{*}}$, Perry E Radau ${ }^{1}$ \\ From 16th Annual SCMR Scientific Sessions \\ San Francisco, CA, USA. 31 January - 3 February 2013
}

\begin{abstract}
Background
Previously a myocardial tissue classification algorithm has been developed to locate and quantify infarct in a given myocardial region-of-interest specified on late gadolinium enhancement (LGE) MR images [1]. To complete the automation requires an endocardial and epicardial contour detection algorithm to replace the current practice of manual contouring that is time-consuming and subject to intra- and inter-observer variability. Challenges include: 1) the intensity inhomogeneity of both the healthy and infarct myocardium; 2) the existence of an infarct on a given slice is not known a priori; 3 ) a subendocardial infarct region's boundary can be easily mistaken for the endocardial contour due to the proximity and strength of the edge (gradient); and 4) incorporating prior anatomical information (e.g., cine steady-state free precession (SSFP) MRI) while allowing for possible motion between separate studies.
\end{abstract}

\section{Methods}

The proposed deformable contour algorithm addresses these challenges by minimizing an energy function that: 1) incorporates intensity overlap into the deformable model by applying Bhattacharyya coefficient [2]; 2) eliminates the image gradient term of the energy function; and 3) incorporates hard constraints based upon prior information about contour shape and myocardium thickness from the pre-delineated contours of the corresponding cine SSFP image (see equation in Figure 1). Ten patients with known CAD and chronic MI had cardiac LGE MR scans. Our method uses pre-delineated endoand epicardial contours from the corresponding cine MRI as a priori knowledge. We registered the corresponding cine MR image to the LGE image using mutual information image registration with affine transformation, then applied the resulting transform to the pre-delineated endo- and epicardial cine MRI contours, to initialize the LGE MRI segmentation. The contours were refined by minimizing the deformable contour energy function locally with greedy optimization [3].

\section{Results}

The average perpendicular distance between manually drawn and automatically detected contours was 1.03 $\pm 0.4 \mathrm{~mm}$ and $0.92 \pm 0.32 \mathrm{~mm}$ for endocardial and epicardial contours, respectively. The average Dice coefficient was $0.95 \pm 0.02$ for endocardium and $0.97 \pm 0.01$ for epicardium and $0.85 \pm 0.05$ for the myocardium. Representative results in Figure 2. By visual examination 19\% contours (27/144 contours) required manual corrections.

$$
\min _{C}\left\{E(C, I)=\lambda_{1} \oint\left|C^{\prime}(s)\right| d s+\lambda_{2} f\left|C^{\prime \prime}(s)\right| d s+\frac{\lambda_{3}}{R_{B}} \int_{B}|I(x, y)-\bar{B}| d x d y+\frac{\lambda_{4}}{|\bar{M}-\bar{B}|}+\lambda_{5} \times B a(P(B) / P(M))\right\}
$$

Figure 1 Energy function of the deformable model. $C(s)=(x(s), y(s))$ is the parameterized contour (endo- and epicardial), $P(B)$ is the histogram of blood pool and $P(M)$ as the histogram of myocardium. $B a(f / g)$ is the Bhattacharyya coefficient measuring the amount of overlap between two statistical samples $f$ and $g$. Shape is the Fourier descriptors of the discretized contour. Each contour is constrained by the corresponding cine contour shape, and by myocardial thickness.

${ }^{1}$ Imaging Research, Sunnybrook research institute, Toronto, ON, Canada

Full list of author information is available at the end of the article

(c) 2013 Lu et al; licensee BioMed Central Ltd. This is an Open Access article distributed under the terms of the Creative Commons Attribution License (http://creativecommons.org/licenses/by/2.0), which permits unrestricted use, distribution, and reproduction in any medium, provided the original work is properly cited. 

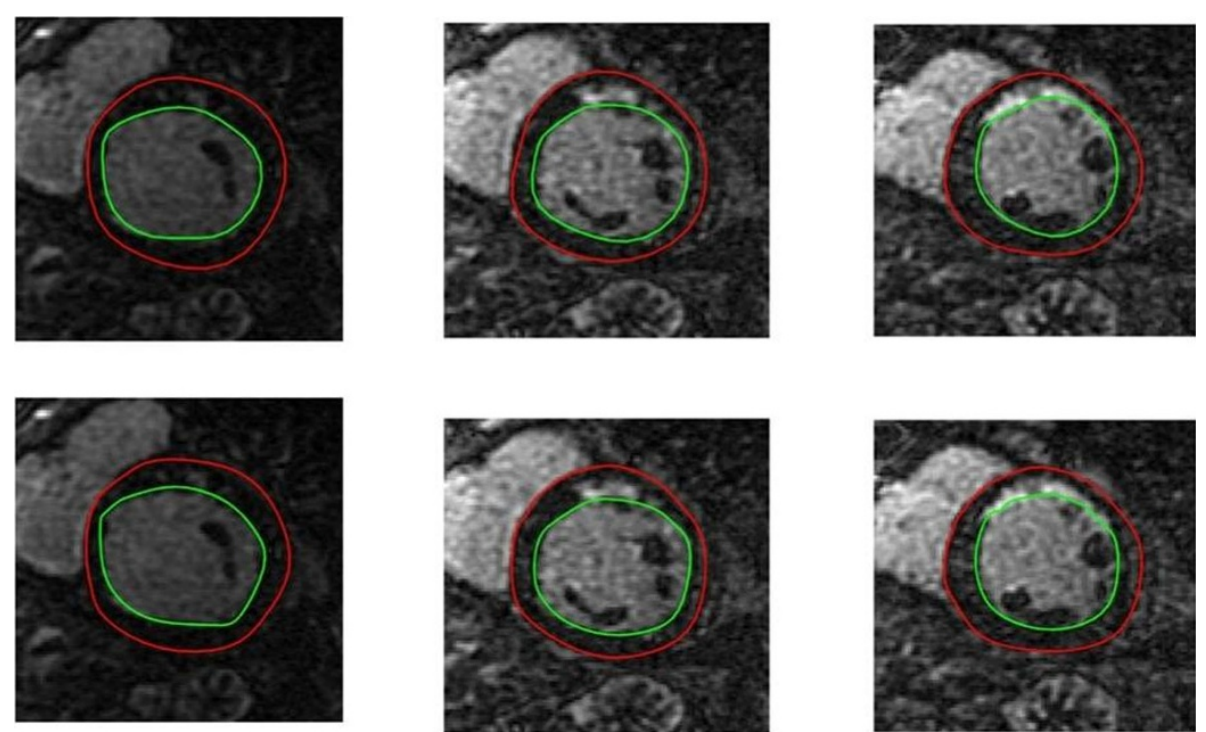

Figure 2 Representative automatic segmentation results (top row) compared to expert results (bottom row).

This is a substantial improvement compared with previous rate of about $50 \%$ when propagating cine contours without the deformable contour algorithm.

\section{Conclusions}

Our method provides a high degree of automation and accuracy. The results for the proposed automated segmentation technique indicate that it will streamline accurate quantification of myocardial infarct on LGE MR infarct images in clinical practice.

\section{Funding}

MaRS Innovation 2011 Medical Sciences Competitive Proof of Principle (MSCPoP) for Medical Devices and Ontario Research Fund, (Imaging for Cardiovascular Therapeutics).

\section{Author details}

${ }^{1}$ Imaging Research, Sunnybrook research institute, Toronto, ON, Canada.

${ }^{2}$ Medical Biophysics, University of Toronto, Toronto, ON, Canada.

\section{Published: 30 January 2013}

\section{References}

1. Lu YL, et al: Automated quantification of myocardial infarction using graph cuts on contrast delayed enhanced magnetic resonance images. Quantitative Imaging in Surgery and Medicine 2012, 2:81-6.

2. Williams DJ, et al: A fast algorithm for active contours and curvature estimation. CVGIP: Image Understanding 1992, 55:14-26.

3. Comaniciu $D$, et al: Real-time tracking of non-rigid objects using mean shift. IEEE conference on Computer Vision and Pattern Recognition 2000, 2:142-9.

doi:10.1186/1532-429X-15-S1-P14

Cite this article as: Lu et al: Automatic myocardium segmentation of LGE MRI by deformable models with prior shape data. Journal of Cardiovascular Magnetic Resonance 2013 15(Suppl 1):P14.

\section{Submit your next manuscript to BioMed Central} and take full advantage of:

- Convenient online submission

- Thorough peer review

- No space constraints or color figure charges

- Immediate publication on acceptance

- Inclusion in PubMed, CAS, Scopus and Google Scholar

- Research which is freely available for redistribution
Biomed Central 